\& Research Square

\title{
Phenotypic Characterization of Extended Spectrum Beta-lactamase, Class C Cephalosporinase and Carbapenemase-Producing Klebsiella Species Isolated from Patients Consulted at Four Yaounde-Based Hospitals.
}

Emilia Enjema Lyonga Mbamyah ( $\nabla$ emilialyo@yahoo.co.uk)

the University of Yaounde 1

Mangum Patience Kumcho

Catholic University of Central Africa

Michel Toukam

the University of Yaounde 1

Dieudonné Sedena

University of Buea

Florence Anjabie Enyeji

the University of Yaounde 1

Aime-Caesar Teukam

the University of Yaounde 1

Modestine Djuissi

the University of Yaounde 1

Martha Tongo Mesembe

the University of Yaounde 1

George Mondinde Ikomey

the University of Yaounde 1

Anicette Chafa Betbeui

Yaounde University Teaching Hospital

William Baiye

Yaounde University Teaching Hospital

Agnes Bedie Eyoh

the University of Yaounde 1

Hortense Kamga Gonsu

the University of Yaounde 1

Research Article

Keywords: Klebsiella spp., multidrug resistance, ESBL, AmpC, Carbapenemase

Posted Date: August 12th, 2021

DOI: https://doi.org/10.21203/rs.3.rs-793227/v1

License: (c) (i) This work is licensed under a Creative Commons Attribution 4.0 International License. Read Full License 


\section{Abstract}

Background: Klebsiella spp. are bacteria of medical importance for their role in opportunistic infections. These infections are often difficult to treat because of acquired resistance to one or several families of antimicrobials. The present study aimed at detecting Extended Spectrum Beta-lactamase (ESBL), Class C cephalosporinase (AmpC) and carbapenemase resistant phenotypes of Klebsiella spp. isolated from patients consulted at four Yaounde-based hospitals.

Results: The frequency of the species isolated was Klebsiella pneumoniae (69\%), K. oxytoca (14\%), K. ozaenae (12\%) and K. rhinoscleromatis (5\%). Isolates were most resistant to penicillins ( $90 \%)$, sulphonamides (84\%), cepaholosporins ( $80 \%)$, and least resistant to carbapenems (10.2\%). Three isolates namely: two K. oxytoca and one K. pneumoniae were resistant to all twenty-eight (28) antibiotics tested. Klebsiella pneumoniae was the species with the most multidrug resistant isolates (59.4\%). Most isolates (83.6\%) expressed at least one resistance phenotype, while $63.6 \%$ of the isolates expressed all three phenotypes. Many of the isolates were ESBL producers (71.6\%), while fewer isolates were carbapenemase (26.7\%) and AmpC (6.6\%) producers. Three carbapenemases (Klebsiella pneumoniae carbapenemase-KPC, Metallo-Beta Lactamase-MBL and OXA-48) were detected from $26.7 \%$ of the isolates and the combination KPC and MBL were the most detected phenotypes (12.9\%).

Conclusion: These results reveal that resistance of Klebsiella spp. to cephalosporins is high and this may be exacerbated as a result of the co-expression of $\mathrm{AmpC}$ and carbapenemases. About a quarter of the isolates had acquired carbapenemases that confer resistance to all beta-lactamases and carbapenems which constitute last line drugs. The resistance burden is further strengthened in isolates that acquired more than one carbapenemase aggravating associated patient morbidity and mortality. Therefore, it is necessary to continue monitoring antimicrobial resistance of local strains for better informed decisions on empirical treatment guide and better patient care.

\section{Background}

Klebsiella species are bacteria responsible for community and hospital acquired infections (1). The emergence and global spread of Klebsiella species harbouring Klebsiella pneumoniae carbapenemase (KPC), Metallo- $\beta$-lactamases (MBL) and even ESBL, confer resistance to several antibiotics. This threatens effective control and treatment of ensuing invasive infections (2), as such patient outcome is characterized by high mortality (3). Antimicrobial resistance (AMR) complicates treatment achieved through early use of empirical antibiotics in parts of the world, where the bulk of health problems are caused by infectious disease, posing big challenges for public health.

Klebsiella pneumoniae and to an extent $K$. oxytoca are the species that pose the most problem in terms of AMR. Enzymes (ESBL, carbapenemases), horizontal gene transfer (HGT) and other resistance mechanisms are responsible for extensive AMR determinants in pathogens (4). The burden of AMR due to Klebsiella spp. and the putative resistance phenotypes responsible may not be fully appreciated in Cameroon because of paucity of data (5). AMR surveillance has gaps because the capacity for early detection of AMR and surveillance of infections caused by priority pathogens is not sufficient. Only few laboratories have the capacity to identify pathogens, detect resistance profiles because of an inadequacy of technical platforms and training (6). Previous studies from Cameroon on bacteria resistance to certain families of antibiotics commonly used to treat infections caused by Gram negative bacteria such as $\beta$-lactams, derivatives (cephalosporins and carbapenems) and quinolones, underscore a high prevalence of drug resistance among some WHO high priority Enterobacteriaceae(7) (8) (9). Antimicrobial susceptibility testing revealed a generally high resistance to first, second and third generation cephalosporins and low resistance to carbapenems. Phenotypic and genotypic characterization of resistance revealed a high prevalence of ESBL (CTX-M, SHV-2, AmpC)(10) (8) and the presence of plasmid mediated quinolone resistant genes ( $q n r B$ and $q n r S$ )(9). Constant monitoring and identification of resistance phenotypes is necessary for the purpose of infection control and choosing empiric treatment.

\section{Results:}

The ages of the participants ranged from 0 to 95 years, with a mean of 32.6 years. The $0-9$ years age group was most represented and accounted for $24.4 \%$ $(55 / 225)$ of isolates. The majority of participants were hospitalized patients $57 \%$ (129/225). Klebsiella species were isolated from thirteen (13) clinical specimens. Urine was the specimen that was the most frequently analysed accounting for $42.7 \%(96 / 225)$ of the isolates. The frequency of the species isolated was Klebsiella pneumoniae (69\%), K. oxytoca (14\%), K. ozaenae (12\%) and K. rhinoscleromatis (5\%).

Resistance of isolates to amoxicillin was highest with a frequency of $99.1 \%(223 / 225)$ and similarly very high resistance rates were recorded for amoxicillin clavulanate (93.3\%), ticarcillin (95.1\%), ticarcillin clavulanate (83.1\%) and piperacillin (86.2\%). The action of the inhibitor tazobactam improved the activity of piperacillin with a resulting moderate resistance rate of $39.1 \%$. Resistance to cephalosporins was very high against first generation (cefalotin $84.9 \%$ ) and second generation cephalosporins (cefuroxim 82.2\%). The resistance of isolates to third generation cephalosporins was high (ceftriaxone-76.4\%, ceftazidime-75.1\% and cefotaxime-54.7\%) and equally as high to the lone fourth generation cephalosporin tested (cefepime-70.2\%) and the lone monobactam tested (aztreonam-68.4\%). Carbapenems were the most sensitive drugs with the lowest resistance frequencies of $10.7 \%$ and $10.2 \%$ for meropenem and imipenem respectively. The only sulphonamide tested was sulphamethoxazole trimethoprim (84.0\%), which had a resistance frequency comparable to that of penicillins (ticarcillin and piperacillin).

Four aminoglycosides were tested and their resistance profiles were diverse. Although the antibiotic resistance rate was generally high within the class (gentamycin-61.8\%, tobramycin-56.9\% and netilmicin 38.2\%), amikacin was the exception with a low resistance rate of $14.7 \%$.

The resistance to quinolones was fairly high compared to the penicillins, cephalosporins and monobactam. Isolates were most resistant to pipemedic acid (53.8\%) and least resistant to levofloxacin (33.8\%). Tables 1 and 2 in the supplementary file highlight these results. 
Table 1

Antimicrobial susceptibility profile of isolates to all classes of antibiotics tested

\begin{tabular}{|c|c|c|c|c|c|c|c|}
\hline \multirow{2}{*}{\multicolumn{2}{|c|}{$\begin{array}{l}\text { Antibiotic Antibiotics } \\
\text { Family }\end{array}$}} & \multicolumn{2}{|l|}{ Intermediate } & \multicolumn{2}{|l|}{ Resistant } & \multicolumn{2}{|l|}{ Sensitive } \\
\hline & & Frequency & $\%$ & Frequency & $\%$ & Frequency & $\%$ \\
\hline \multirow[t]{15}{*}{ Beta lactams } & Amoxicillin & 0 & $0 \%$ & 223 & 2 & $0.9 \%$ & \\
\hline & Amoxicillin clavulanate & 1 & $0.4 \%$ & 210 & 14 & $6.2 \%$ & \\
\hline & Ticarcillin & 4 & $1.8 \%$ & 214 & 7 & $3.1 \%$ & \\
\hline & Ticarcillin clavulanate & 15 & $6.7 \%$ & 187 & 23 & $10.2 \%$ & \\
\hline & Piperacillin & 15 & $6.7 \%$ & 194 & 16 & $7.1 \%$ & \\
\hline & Piperacillin tazobactam & 22 & $9.8 \%$ & 88 & $39.1 \%$ & 115 & $51.1 \%$ \\
\hline & Cefalotin & 6 & $2.7 \%$ & 191 & 28 & $12.4 \%$ & \\
\hline & Cefuroxime & 2 & $0.9 \%$ & 185 & 38 & $16.9 \%$ & \\
\hline & Ceftazidime & 21 & $9.3 \%$ & 169 & 35 & $15.6 \%$ & \\
\hline & Ceftriaxone & 7 & $3.1 \%$ & 172 & 46 & $20.4 \%$ & \\
\hline & Cefotaxime & 14 & $6.2 \%$ & 123 & $54.7 \%$ & 88 & $39.1 \%$ \\
\hline & Cefepime & 13 & $5.8 \%$ & 158 & 54 & $24 \%$ & \\
\hline & Aztreonam & 12 & $5.3 \%$ & 154 & $68.4 \%$ & 59 & $26.2 \%$ \\
\hline & Sulphonamide & Sulfamethaxazole & 2 & $0.9 \%$ & 189 & 34 & $15.1 \%$ \\
\hline & \multirow[t]{4}{*}{ Aminoglycosides } & Gentamicin & 5 & $2.2 \%$ & 139 & $61.8 \%$ & 81 \\
\hline Amikacin & & 4 & $1.8 \%$ & 33 & $14.7 \%$ & 188 & $83.6 \%$ \\
\hline Tobramycin & & 23 & $10.2 \%$ & 128 & $56.9 \%$ & 74 & $32.9 \%$ \\
\hline Netilmicin & & 13 & $5.8 \%$ & 86 & $38.2 \%$ & 126 & $56 \%$ \\
\hline \multirow[t]{8}{*}{ Quinolones } & NalidixicAcid & 14 & $6.2 \%$ & 90 & $40 \%$ & 121 & $53.8 \%$ \\
\hline & PipemidicAcid & 12 & $5.3 \%$ & 121 & $53.8 \%$ & 92 & $40.9 \%$ \\
\hline & Ciprofloxacin & 20 & $8.9 \%$ & 90 & $40 \%$ & 115 & $51.1 \%$ \\
\hline & Norfloxacin & 10 & $4.4 \%$ & 78 & $34.7 \%$ & 137 & $60.9 \%$ \\
\hline & Levofloxacin & 12 & $5.3 \%$ & 76 & $33.8 \%$ & 137 & $60.9 \%$ \\
\hline & Ofloxacin & 12 & $5.3 \%$ & 90 & $40 \%$ & 123 & $54.7 \%$ \\
\hline & Gatifloxacin & 13 & $5.8 \%$ & 98 & $43.6 \%$ & 114 & $50.7 \%$ \\
\hline & Mixofloxacin & 14 & $6.2 \%$ & 100 & $44.4 \%$ & 111 & $49.3 \%$ \\
\hline
\end{tabular}


Table 2

Comparism of the resistance profile amid hospitalized and outpatients.

\begin{tabular}{|c|c|c|c|c|c|}
\hline \multirow{2}{*}{$\begin{array}{l}\text { Beta lactams } \\
\text { Hospitalized patient }\end{array}$} & \multicolumn{5}{|c|}{ Patient category } \\
\hline & & Out-patient & & p-value & \\
\hline Frequency & $\%$ & Frequency & $\%$ & & \\
\hline Amoxicillin & 128 & $99.2 \%$ & 95 & $99 \%$ & 0.833 \\
\hline Ticarcillin & 126 & $97.7 \%$ & 88 & $91.7 \%$ & 0.045 \\
\hline Piperacillin & 118 & $91.5 \%$ & 76 & $79.2 \%$ & 0.007 \\
\hline Cefalotin & 119 & $92.2 \%$ & 72 & $75 \%$ & 0.002 \\
\hline Cefotaxime & 73 & $56.6 \%$ & 50 & $52.1 \%$ & 0.737 \\
\hline Cefepime & 97 & $75.2 \%$ & 61 & $63.5 \%$ & 0.166 \\
\hline Aztreonam & 4 & $3.1 \%$ & 5 & $5.2 \%$ & 0.393 \\
\hline Imipenem & 9 & $7 \%$ & 14 & $14.6 \%$ & 0.115 \\
\hline Sulfamethoxazole Trimethoprim & 116 & $89.9 \%$ & 73 & $76 \%$ & 0.018 \\
\hline \multicolumn{6}{|l|}{ Aminoglycosides } \\
\hline Tobramycin & 80 & $62 \%$ & 48 & $50 \%$ & 0.021 \\
\hline \multicolumn{6}{|l|}{ Quinolones } \\
\hline Nalidixic acid & 55 & $42.6 \%$ & 35 & $36.5 \%$ & 0.6 \\
\hline Pipemidic acid & 75 & $58.1 \%$ & 46 & $47.9 \%$ & 0.281 \\
\hline Ciprofloxacin & 59 & $45.7 \%$ & 31 & $32.3 \%$ & 0.124 \\
\hline Norfloxacin & 52 & $40.3 \%$ & 26 & $27.1 \%$ & 0.085 \\
\hline Levofloxacin & 49 & $38 \%$ & 27 & $28.1 \%$ & 0.193 \\
\hline Ofloxacin & 59 & $45.7 \%$ & 31 & $32.3 \%$ & 0.07 \\
\hline Mixofloxacin & 65 & $50.4 \%$ & 35 & $36.5 \%$ & 0.092 \\
\hline
\end{tabular}


Table 3

Comparism of antibiotic resistance profile amid bacteria species

\begin{tabular}{|c|c|c|c|c|c|c|c|c|c|}
\hline & \multicolumn{2}{|c|}{ Klebsiella oxytoca } & \multicolumn{2}{|c|}{ Klebsiella ozaenae } & \multicolumn{2}{|c|}{ Klebsiella pneumoniae } & \multicolumn{2}{|c|}{ Klebsiella minoscleromatis } & \multirow[t]{2}{*}{$p$-value } \\
\hline & Count & $\%$ & Count & $\%$ & Count & $\%$ & Count & $\%$ & \\
\hline \multicolumn{10}{|l|}{ Beta lactams } \\
\hline Amoxicillin & 32 & $100 \%$ & 25 & $96.2 \%$ & 154 & $99.4 \%$ & 12 & $100 \%$ & 0.379 \\
\hline Ticarcillin & 32 & $100 \%$ & 22 & $84.6 \%$ & 149 & $96.1 \%$ & 11 & $91.7 \%$ & 0.058 \\
\hline Ticarcillin clavulanate & 29 & $90.6 \%$ & 19 & $73.1 \%$ & 128 & $82.6 \%$ & 11 & $91.7 \%$ & 0.646 \\
\hline Piperacillin & 29 & $90.6 \%$ & 21 & $80.8 \%$ & 133 & $85.8 \%$ & 11 & $91.7 \%$ & 0.771 \\
\hline Piperacillin tazobactam & 14 & $43.8 \%$ & 9 & $34.6 \%$ & 63 & $40.6 \%$ & 2 & $16.7 \%$ & 0.728 \\
\hline Cefalotin & 30 & $93.8 \%$ & 19 & $73.1 \%$ & 130 & $83.9 \%$ & 12 & $100 \%$ & 0.217 \\
\hline Cefuroxime & 28 & $87.5 \%$ & 22 & $84.6 \%$ & 124 & $80 \%$ & 11 & $91.7 \%$ & 0.076 \\
\hline Ceftazidime & 23 & $71.9 \%$ & 16 & $61.5 \%$ & 119 & $76.8 \%$ & 11 & $91.7 \%$ & 0.139 \\
\hline Ceftriaxone & 22 & $68.8 \%$ & 16 & $61.5 \%$ & 123 & $79.4 \%$ & 11 & $91.7 \%$ & 0.136 \\
\hline Cefotaxime & 18 & $56.3 \%$ & 10 & $38.5 \%$ & 90 & $58.1 \%$ & 5 & $41.7 \%$ & 0.315 \\
\hline Cefepime & 20 & $62.5 \%$ & 16 & $61.5 \%$ & 112 & $72.3 \%$ & 10 & $83.3 \%$ & 0.302 \\
\hline Aztreonam & 19 & $59.4 \%$ & 15 & $57.7 \%$ & 111 & $71.6 \%$ & 9 & $75 \%$ & 0.531 \\
\hline Meropenem & 7 & $21.9 \%$ & 2 & $7.7 \%$ & 15 & $9.7 \%$ & 0 & $0 \%$ & 0.09 \\
\hline \multicolumn{10}{|l|}{ Sulphonamide } \\
\hline Sulfamethaxazole Trimethoprim & 27 & $84.4 \%$ & 23 & $88.5 \%$ & 128 & $82.6 \%$ & 11 & $91.7 \%$ & 0.752 \\
\hline \multicolumn{10}{|l|}{ Aminoglycosides } \\
\hline Gentamicin & 20 & $62.5 \%$ & 15 & $57.7 \%$ & 95 & $61.3 \%$ & 9 & $75 \%$ & 0.757 \\
\hline Amikacin & 6 & $18.8 \%$ & 4 & $15.4 \%$ & 23 & $14.8 \%$ & 0 & $0 \%$ & 0.722 \\
\hline Tobramycin & 16 & $50 \%$ & 18 & $69.2 \%$ & 85 & $54.8 \%$ & 9 & $75 \%$ & 0.157 \\
\hline Netilmicin & 13 & $40.6 \%$ & 14 & $53.8 \%$ & 55 & $35.5 \%$ & 4 & $33.3 \%$ & 0.595 \\
\hline \multicolumn{10}{|l|}{ Quinolones } \\
\hline Nalidixic acid & 16 & $50 \%$ & 12 & $46.2 \%$ & 59 & $38.1 \%$ & 3 & $25 \%$ & 0.304 \\
\hline Ciprofloxacin & 13 & $40.6 \%$ & 12 & $46.2 \%$ & 62 & $40 \%$ & 3 & $25 \%$ & 0.638 \\
\hline Norfloxacin & 13 & $40.6 \%$ & 11 & $42.3 \%$ & 52 & $33.5 \%$ & 2 & $16.7 \%$ & 0.778 \\
\hline Levofloxacin & 13 & $40.6 \%$ & 11 & $42.3 \%$ & 48 & $31 \%$ & 4 & $33.3 \%$ & 0.669 \\
\hline Ofloxacin & 15 & $46.9 \%$ & 12 & $46.2 \%$ & 59 & $38.1 \%$ & 4 & $33.3 \%$ & 0.825 \\
\hline Gatifloxacin & 15 & $46.9 \%$ & 12 & $46.2 \%$ & 68 & $43.9 \%$ & 3 & $25 \%$ & 0.497 \\
\hline Mixofloxacin & 16 & $50 \%$ & 13 & $50 \%$ & 66 & $42.6 \%$ & 5 & $41.7 \%$ & 0.692 \\
\hline
\end{tabular}

Three isolates from hospitalized patients were resistant to all 28 antibiotics tested (super bugs). One $K$. pneumoniae and two $K$. oxytoca. The isolates were from three different clinical specimens namely: cereobrospinal fluid, wounds and catheter drain. The isolates tested expressed several resistance enzymes namely: Extended spectrum $\beta$-lactamases, AmpC and carbapenamases. These results are presented in Table 4 
General profile of resistance phenotype

\begin{tabular}{|c|c|c|c|}
\hline Classes of phenotypes & Phenotypes & Frequency & $\%$ \\
\hline \multirow[t]{4}{*}{ Extended spectrum beta-lactamase with variations $(\mathrm{ESBL})(\mathrm{N}=161)$} & ESBL & 143 & $63.6 \%$ \\
\hline & $\mathrm{ESBL}, \mathrm{AmpC}$ & 11 & $4.9 \%$ \\
\hline & ESBL, porin loss & 7 & $3.1 \%$ \\
\hline & None & 64 & $28.4 \%$ \\
\hline \multirow[t]{4}{*}{ Class $C$ cephalosporinases with variations $(N=15)$} & AmpC & 3 & $1.3 \%$ \\
\hline & AmpC, ESBL & 11 & $4.9 \%$ \\
\hline & AmpC, KPC and MBL & 1 & $0.4 \%$ \\
\hline & None & 210 & $93.3 \%$ \\
\hline \multirow[t]{6}{*}{ Carbapenamases with variations $(\mathrm{N}=60)$} & KPC & 9 & $4 \%$ \\
\hline & $\mathrm{KPC}, \mathrm{MBL}$ & 29 & $12.9 \%$ \\
\hline & AmpC, KPC and MBL & 1 & $0.4 \%$ \\
\hline & MBL & 4 & $1.8 \%$ \\
\hline & OXA-48 & 17 & $7.6 \%$ \\
\hline & None & 165 & $73.3 \%$ \\
\hline
\end{tabular}

There was a high frequency of isolates which expressed resistance phenotypes or mechanisms (83.6\% -188/225) compared with $16.4 \%$ (37/225) of the isolates that did not express any resistance phenotype.

A majority of the isolates were ESBL producers (63.6\%), while ESBL in combination with porin loss and AmpC were produced by $71.6 \%$ (161/225) of isolates. Class $\mathrm{C}$ cephalosporinases alone were produced by $1.3 \%(3 / 225)$ of isolates while in combination with other enzymes they were produced by $6.7 \%$ ( $15 / 225)$. The isolates produced several carbapenamases either as sole enzyme or in multiple enzyme combinations resulting in a total of $26.7 \%$ of the isolates being carbapenamase producers. The most frequently produced carbapenamases were the combination of KPC and MBL enzymes (12.9\%). These results are highlighted on Tables 4 and 5 .

Table 5

Comparism of resistance phenotypes per species

\begin{tabular}{|c|c|c|c|c|c|c|}
\hline Classes of phenotypes & Phenotypes & $\begin{array}{l}\text { Klebsiella } \\
\text { oxytoca }\end{array}$ & $\begin{array}{l}\text { Klebsiella } \\
\text { ozaenae }\end{array}$ & $\begin{array}{l}\text { Klebsiella } \\
\text { rhinoscleromatis }\end{array}$ & p-value & \\
\hline \multirow{4}{*}{$\begin{array}{l}\text { Extended spectrum beta-lactamase with variations } \\
(\mathrm{ESBL})(\mathrm{N}=161)\end{array}$} & ESBL & $53.8 \%$ & $66.5 \%$ & $83.3 \%$ & \multirow[t]{4}{*}{0.470} & \\
\hline & $\mathrm{ESBL}, \mathrm{AmpC}$ & $6.3 \%$ & $7.7 \%$ & $4.5 \%$ & & $0 \%$ \\
\hline & ESBL, porin loss & $3.1 \%$ & $0 \%$ & $3.9 \%$ & & $0 \%$ \\
\hline & None & $40.6 \%$ & $38.5 \%$ & $25.2 \%$ & & $16.7 \%$ \\
\hline \multirow{4}{*}{$\begin{array}{l}\text { Class } C \text { cephalosporinases }(\mathrm{AmpC}) \text { with variations } \\
(\mathrm{N}=15)\end{array}$} & AmpC & $6.3 \%$ & $0.0 \%$ & $0.6 \%$ & $0 \%$ & \multirow[t]{4}{*}{0.468} \\
\hline & AmpC, ESBL & $6.3 \%$ & $7.7 \%$ & $4.5 \%$ & $0 \%$ & \\
\hline & $\begin{array}{l}\text { AmpC, KPC and } \\
\text { MBL }\end{array}$ & $0 \%$ & $0 \%$ & $0.6 \%$ & $0 \%$ & \\
\hline & None & $87.5 \%$ & $92.3 \%$ & $94.2 \%$ & $100 \%$ & \\
\hline \multirow[t]{6}{*}{ Carbapenamases with variations $(\mathrm{N}=60)$} & KPC & $7.7 \%$ & $2.6 \%$ & $0 \%$ & \multirow[t]{6}{*}{0.579} & \\
\hline & $\mathrm{KPC}, \mathrm{MBL}$ & $7.7 \%$ & $12.9 \%$ & $8.3 \%$ & & \\
\hline & $\begin{array}{l}\text { AmpC, KPC, and } \\
\text { MBL }\end{array}$ & $0 \%$ & $0 \%$ & $0 \%$ & & \\
\hline & MBL & $0 \%$ & $0 \%$ & $2.6 \%$ & & $0 \%$ \\
\hline & OXA-48 & $9.4 \%$ & $0 \%$ & $9 \%$ & & $0 \%$ \\
\hline & None & $62.6 \%$ & $84.6 \%$ & $72.3 \%$ & & $91.7 \%$ \\
\hline
\end{tabular}

\section{Discussion:}


Our findings revealed that the predominant species was Klebsiella pneumoniae (69\%). Current evidence suggests that $K$. pneumoniae is the most prevalent Klebsiella species due to its wide-ranging ecological distribution, considerably more varied DNA composition that facilitates adaptation to diversed environments, greater AMR gene diversity and plasticity and a higher plasmid burden than other Gram negative opportunists (14). Therefore, K. pneumoniae is better organized for mobilizing resistant genes from other drug resistant bacteria in the environment or in animal/human microbial communities rendering otherwise non-resistant strains multidrug resistant. Isolates were most recovered from urine 42.7\% (96/225) confirming that urinary tract infections (UTIs) are among the most common infections both in hospital and community infections in Cameroon (15). In a bid to treat common infections, widespread inappropriate and disproportionate use of antimicrobials has led to the emergence of multidrug resistant isolates of Klebsiella spp. making such infections more difficult to treat(16).

As expected, resistance of isolates to amoxicillin was highest $99.1 \%(223 / 225)$ likewise, amoxicillin clavulanate (93.3\%), ticarcillin (95.1\%), ticarcillin clavulanate (83.1\%) and piperacillin (86.2\%). It is has been demonstrated that $K$. pneumoniae has intrinsic resistance to penicillin $\mathrm{G}$ and ampicillin due to the presence of a Class A SHV-1 penicillinase. However, the resistance of isolates to penicillin inhibitors like clavulanate and tazobactam that normally restore the activity of penicillins was also very high in our study as observed in amoxicillin clavulanate (93.3\%), ticarcillin clavulanate (83.1\%) and piperacillin (86.2\%). These results contrast results within the same setting (17) which reported resistance rates of $39 \%$ and $22 \%$ respectively to amoxicillin clavulanate and ticarcillin clavulanate and $38 \%$ while piperacillin tazobactam $39.1 \%$ was comparable to the present study. Such high rates of resistance to penicillins and penicillin inhibitor combinations can be further explained by enhanced resistance mechanisms co-expressed by a good number of the isolates such as ESBL (71.6\%) on the one hand that hydrolyze penicillins while AmpC and carbapenemases hydrolyze ESBL inhibitors like clavulanate.

Resistance rates to first generation cephalosporins (cefalotin $84.9 \%$ ), second generation cephalosporins (cefuroxim $82.2 \%$ ), third generation cephalosporins (ceftriaxone-76.4\%. ceftazidime-75.1\% and cefotaxime-54.7\%) and likewise fourth generation cephalosporin (cefepime-70.2\%) and a monobactam (aztreonam-68.4\%) was higher than those earlier reported (17) (cefalotin $60 \%$, ceftazidime $51 \%$, cefotaxime $51 \%$, cefepime $26 \%$ and aztreonam $45 \%$ ). This indicates that within our study setting, there has been more than $20 \%$ increase in resistance to all generations of cephalosporins since 2015 . Resistance to cephalosporins is mainly as a result of the production of Extended Spectrum $\beta$-Lactamases (ESBL). They hydrolyze penicillins, first, second, third, fourth generation, monobactams but not cephamycins (cefoxitin), beta-lactam inhibitors and carbapenems. Though there maybe large variations in geographical distribution of ESBL genes due to differences in antibiotic use, hygiene and co-expression of other virulence factors such as efflux pumps, siderophores, polysaccharide capsule proteins and fimbriae (1), what cuts across the studies cited above is that there is a global rise in resistance to cephalosporins. This is most likely because of clonal expansion and an increase in dissemination of ESBL genes through horizontal gene transfer mechanisms by plasmids or de novo mutations. In our study, $71.6 \%$ of isolates were ESBL producers (63.6\% ESBL alone, $4.9 \% \mathrm{ESBL}$ and AmpC, 3.1\% ESBL with porin loss).

Ambler Class $\mathrm{C}$ cephalosporinases (AmpC) are naturally produced by some Enterobacteriaceae like E. coli, Shigella spp., P. aeruginosa and Enterobacter spp. but not Klebsiella spp. which are known to have acquired mobile AmpC genes (CMY-2 and DHA-1) through HGT mechanisms. AmpCs hydrolyze penicilins, third generation cephalosporins, monobactams but not fourth generation cephalosporins, carbapenems and they are poorly inhibited by ESBL inhibitors like clavulanate (13). Their overall frequency has remained comparatively far below that of ESBL in most studies (1),(8), (18). In this study (15/225) 6.6\% of isolates were $\mathrm{AmpC}$ producers (1.3\% AmpC alone, $4.9 \% \mathrm{AmpC}$ and $\mathrm{ESBL}, 0.4 \% \mathrm{AmpC}+\mathrm{MBL}+\mathrm{KPC}$ ). It is not uncommon for AmpC producers to co-express other enzymes. This was mostly the case among hospitalized patients and within the species $K$. pneumoniae and $K$. oxytoca. Prolonged hospitalization and intensive use of antibiotics within the hospital seems to increase selection pressure of multidrug resistant isolates, implicitly such infections are more difficult to treat. Carbapenems constitute one of the last treatment options for serious infections. Resistance to carbapenems is a serious call for concern because carbapenemases confer resistance to all beta-lactam drugs rendering ensuing infections associated with high morbidity and mortality rates. Furthermore, the carbapenemase genes are easily transferable among Enterobacteriaceae. Since the first case of carbapenemase (KPC) epidemics in America in the mid-1990s, other outbreaks have since occurred as a result of other carbapenemases such as VIM, Oxa-48 and NDM enzymes which have been disseminated globally (14). Whilst, the associated morbidity and mortality as a result of carbapenemase producing bacteria is high, carbapenems are still the most efficient drugs with the lowest levels of drug resistance. In this study, the rate is quite high 26.7\% (60/225) compared to previous reports from our study setting (9), (10), (17). Our values are higher than values by Lyonga-Mbamyah et al., 2020. Their study included all Enterobacteriaceae, thus their values were diluted because some species do not harbour carbapenemase resistant genes as much as some Klebsiella spp. Findings by Betbeui et al., 2015 were carried out only on Klebsiella spp., and they indicate that fives years ago, there was a high resistance rate to carbapenems within our study setting. In Nigeria (19), an incidence of 7.7\% was reported, in Tanzania (15), 32.24\% was reported, in the United Kingdom (1), $0 \%$ was reported and in India (20), it was reported that the trend of carbapenems resistance rose from 7.4 to $84.1 \%$ between 2004 and 2013. In the present study, 23 isolates of 60 carbapenem resistant isolates co-expressed AmpC, ESBL and resistance to quinolones and there was no significant difference between hospitalized and community patients. This implies that carbapenem resistant Klebsiella spp. are not only circulating in hospitals affecting critically ill patients but they are also circulating in our environment complicating treatment for community patients who may resort to auto-medication or empirical treatment without antibiogram results.

\section{Conclusion:}

The frequency of Klebsiella spp. isolates was high. It represented about a quarter of all isolates identified in biological specimens at the collection sites. Isolates were most resistant to penicillins, cephalosporins and sulphamethoxazole trimethoprim. Above three quarters of isolates were resistant to first, second and fourth generation cephalosporins mostly caused by ESBL. The latter resistance may be exacerbated due to co-expression of AmpC and carbapenemase by isolates. The isolates were least resistant to imipenem with slightly over $10 \%$ resistance rate. Though the resistance rate to carbapenems is on the rise, it still remains the most effective drug class. The majority of isolates were ESBL producers, while about a quarter of the isolates were carbapenemase producers and only a few were AmpC producers. One isolate expressed all three resistance phenotypes (ESBL, AmpC, carbapenemase). Three carbapenemases (KPC, MBL and OXA-48) accounted for a quarter of carbapenemase resistance and the combination of KPC and MBL were the most represented phenotypes. Carbapenemases confer resistance to all beta-lactamases. The resistance burden is further strengthened in isolates that acquired 
more than one carbapenemase aggravating associated patient morbidity and mortality. Therefore, it is necessary to continue monitoring antimicrobial resistance of local strains for better informed decisions on empirical treatment guide and better patient care.

\section{Methods}

- Aim of study

The study aimed to detect Extended Spectrum $\beta$-lactamase (ESBL), AmpC and carbapenemase phenotypes of antibiotic resistant Klebsiella spp. isolated from patients consulted at four hospitals in Yaounde.

- Study type and study setting

The study was cross-sectional and descriptive carried out over a period of nine months (February 2020 to November 2020). Isolates were collected from the Yaounde University Teaching Hospital, Yaounde Central Hospital, Yaounde Gynaeco-Obstetric and Paediatric Hospital and the Yaounde General Hospital. Microbiology analysis was carried out at the Centre for the Study and Control of Communicable Diseases, the University of Yaounde I.

- Study population

The study population was that part of the consenting consulted population from whom Klebsiella spp. was isolated from their biological specimens.

- Identification of isolates

Isolates were sub-cultured on eosin methylene blue agar (EMB) purified on nutrient agar and identified using API 20E (Biomerieux, Marcy-l'Etoile France) according to the procedure referenced by the manufacturer (11).

- Antimicrobial susceptibility testing (AST)

Antimicrobial susceptibility testing of twenty eight antibiotics (Rapid Labs Ltd, Colchester ESSEX-UK) from four families was done according to the Kirby Bauer disc diffusion method (12). Escherichia coli ATCC 25922 was used for quality control of the antibiotic discs.

- Detection of ESBL, AmpC and carbapenemases

The screening of ESBL, AmpC and carbapenemase resistance phenotypes was done simultaneously with AST of third generation cephalosporins, cefoxitin and carbapenems respectively. Confirmation of ESBL and AmpC phenotypes was done based on the combination disc diffusion test (CDT) and carbapenemases based on the combination disc synergy test. The detection and confirmation of resistance mechanisms were carried out as per referenced guidelines by the European Union Committee on Antimicrobial Susceptibility Testing (13). The discs were produced by Liofilchem, Roseto degli Abruszzi-Italy and Klebsiella pneumoniae ATCC 700603 was used for quality control of ESBL.

- Statistical analysis

Statistical analysis was done using CS Pro software. The general antimicrobial resistance profile to 28 antimicrobial was tabulated and the resistance phenotypes detected were compared among Klebsiella species, age groups, type of clinical specimen and patient category. A $p$-value less than 0.05 was considered statistically significant.

\section{Declarations}

\section{Ethical consideration}

This research work received ethical approval from the Comité Institutionnel d'Ethique de la Recherche pour la Santé Humaine, Université Catholique d'Afrique Centrale, Ecole des Sciences de la Santéregistered No.2019/020178/CEIRSH/MI and authorizations for research from all four hospitals. Only participants who consented were included in this study.

\section{Consent for publication}

Not applicable

\section{Availability of data and materials}

Data generated or analysed during this study are included in the supplementary information files, the remainder of the data are available from the corresponding author on reasonable request.

\section{Competing interests}

The authors and co-authors have no financial and non-financial competing interests. 


\section{Funding}

This work was carried out with the aid of a grant from UNESCO and the International Development Research Center (IDRC), Ottawa, Canada through the Organization for Women in Science from the Developing World (OWSD). The views expressed herein do not necessarily represent those of UNESCO, IDRC or its Board of Governors.

\section{Authors' contributions}

Emilia Enjema Lyonga Mbamyah conceived the study and designed it together with Mangum Patience Kumcho and Hortense Kamga Gonsu. Emilia Enjema Lyonga Mbamyah, Patience Mangum Kumcho, Florence Anjabie Enyeji, Dieudonne Sedena, Aime-Caesar Teukam, Modestine Djuissi, Agnes Bedie Eyoh, Anicette Chafa Betbeui, William Baiye conducted the laboratory aspect of the study with contributions from Martha Tongo Mesembe and George Mondinde Ikomey. The general supervision was carried out by Emilia Enjema Lyonga Mbamyah. Emilia Enjema Lyonga Mbamyah drafted the article with contributions from Mangum Patience Kumcho and Martha Tongo Mesembe. All the authors reviewed the article. All the authors read and agreed to the final manuscript.

\section{Acknowledgements}

This work was carried out with the aid of a grant from UNESCO and the International Development Research Center (IDRC), Ottawa, Canada. The views expressed herein do not necessarily represent those of UNESCO, IDRC or its Board of Governors.

The authors wish to thank the hospital personnel at the Bacteriology Unit of the Yaounde University Teaching, Yaounde Central Hospital, Yaounde General Hospital and Yaounde Gynaeco-Obstetric and Paediatric Hospital for the services offered during the period of specimen collection and the staff of the Centre for the Study and Control of Communicable Diseases (CSCCD), Faculty of Medicine and Biomedical Sciences, the University of Yaounde1.

\section{References}

1. Moradigaravand D, Martin V, Peacock SJ, Parkhill J. Evolution and Epidemiology of Multidrug-Resistant Klebsiella pneumoniae in the United Kingdom and Ireland. Chiller T, editor. mBio. 2017 Mar 8;8(1):mBio.01976-16, e01976-16.

2. Monitoring progress on antimicrobial resistance [Internet]. [cited 2021 Jun 1]. Available from: https://www.who.int/activities/monitoring-progressantimicrobial-resistance

3. Epstein L, Hunter JC, Arwady MA, Tsai V, Stein L, Gribogiannis M, et al. New Delhi Metallo-- $\beta$-Lactamase-Producing Carbapenem-Resistant Escherichia coli Associated With Exposure to Duodenoscopes. JAMA. 2014 Oct 8;312(14):1447.

4. Hoenigl M, Valentin T, Zarfel G, Wuerstl B, Leitner E, Salzer HJF, et al. Nosocomial Outbreak of Klebsiella pneumoniae Carbapenemase-Producing Klebsiella oxytoca in Austria. Antimicrob Agents Chemother. 2012 Apr;56(4):2158-61.

5. Mouiche MMM, Moffo F, Akoachere J-FTK, Okah-Nnane NH, Mapiefou NP, Ndze VN, et al. Antimicrobial resistance from a one health perspective in Cameroon: a systematic review and meta-analysis. BMC Public Health. 2019 Dec;19(1):1135.

6. Evaluation externe conjointe des principales capacités RSI de la République du Cameroun I MINSANTE [Internet]. [cited 2021 May 5]. Available from: https://www.minsante.cm/site/?q=fr/content/evaluation-externe-conjointe-des-principales-capacit\%C3\%A9s-rsi-de-la-r\%C3\%A9publique-du-cameroun

7. Lonchel Magoué C, Melin P, Gangoué-Piéboji J, Assoumou M-CO, Boreux R, De Mol P. Prevalence and spread of extended-spectrum $\beta$-lactamaseproducing Enterobacteriaceae in Ngaoundere, Cameroon. Clin Microbiol Infect. 2013 Sep;19(9):E416-20.

8. Ebongue CO, Mengue RN, Mefo'o J-PN, Temfack E, Mengue ER, Adiogo D. Phenotypic Detection of Extended Spectrum $\beta$-Lactamase and AmpC producing Enterobacteriaceae Isolated in A General Hospital. J Microbiol Infect Dis. 2018 Sep 15;113-9.

9. et al. Genotypic Diversity and Characterization of Quinolone Resistant Determinants from Enterobacteriaceae in Yaoundé, Cameroon. Open J Med Microbiol. 2020;10(02):33-45.

10. Nouetchognou JS, Ateudjieu J, Jemea B, Mesumbe EN, Mbanya D. Surveillance of nosocomial infections in the Yaounde University Teaching Hospital, Cameroon. BMC Res Notes. 2016 Dec;9(1):505.

11. BioMérieux $\circledast$ sa. Identification system for Enterobacteriaceae and other non-fastidious Gram-negative rods [Internet]. bioMérieux® sa; 2002 [cited 2021 Jun 3]. Available from: http://biomanufacturing.org/uploads/files/587872707301898351-api20einstructions.pdf

12. Bauer AW, Kirby WM, Sherris JC, Turck M. Antibiotic susceptibility testing by a standardized single disk method. Am J Clin Pathol. 1966 Apr;45(4):493-6.

13. EUCAST_detection_of_resistance_mechanisms_170711.pdf [Internet]. [cited 2021 May 5]. Available from: https://www.eucast.org/fileadmin/src/media/PDFs/EUCAST_files/Resistance_mechanisms/EUCAST_detection_of_resistance_mechanisms_170711.pdf

14. Wyres KL, Holt KE. Klebsiella pneumoniae as a key trafficker of drug resistance genes from environmental to clinically important bacteria. Curr Opin Microbiol. 2018 Oct;45:131-9.

15. Mushi MF, Mshana SE, Imirzalioglu C, Bwanga F. Carbapenemase Genes among Multidrug Resistant Gram Negative Clinical Isolates from a Tertiary Hospital in Mwanza, Tanzania. BioMed Res Int. 2014;2014:1-6.

16. Lyonga EE, Toukam M, Nkenfou C, Gonsu HK, Assoumou M-CO, Mesembe MT, et al. Resistance pattern of enterobacteriaceae isolates from urinary tract infections to selected quinolones in Yaoundé. Pan Afr Med J [Internet]. 2015 [cited 2021 May 5];21. Available from: http://www.panafrican-med-

journal.com/content/article/21/105/full/ 
17. Betbeui A, Kamga H, Toukam M, Mbakop C, Lyonga E, Bilong S, et al. Phenotypic Detection of Extended Spectrum Beta-Lactamase and Carbapenemases Produced by Klebsiella spp Isolated from Three Referrals Hospitals in Yaounde, Cameroon. Br Microbiol Res J. 2015 Jan 10;9(1):1-9.

18. Ghotaslou R, Sadeghi MR, Akhi MT, Hasani A, Asgharzadeh M. Prevalence and Antimicrobial Susceptibility Patterns of ESBL, AmpC and Carbapenemase-producing Enterobactericeae Isolated from Hospitalized Patients in Azerbaijan, Iran. Iran J Pharm Res IJPR. 2018;17(Suppl):79-88.

19. Anibijuwon II, Gbala ID, Adebisi OO. Carbapenem-Resistant Enterobacteriaceae among In-Patients of Tertiary Hospitals in Southwest, Nigeria. Not Sci Biol. 2018 Sep 27;10(3):310-7.

20. Odsbu I, Khedkar S, Lind F, Khedkar U, Nerkar S, Orsini N, et al. Trends in Resistance to Extended-Spectrum Cephalosporins and Carbapenems among Escherichia coli and Klebsiella spp. Isolates in a District in Western India during 2004-2014. Int J Environ Res Public Health. 2018 Jan 19;15(1):155. 